\title{
An Effective Strategy to Construct Novel Polyoxometalate-Based Hybrids by Deliberately Controlling Organic Ligand
}

\section{Transformation In Situ}

Table S1. Selected bond lengths $(\AA)$ and angles $\left(^{\circ}\right)$ of compounds 1-4.

\begin{tabular}{|c|c|c|c|}
\hline \multicolumn{4}{|c|}{ Compound 1} \\
\hline $\mathrm{Cu}(1)-\mathrm{N}(3)$ & $1.997(4)$ & $\mathrm{Cu}(1)-\mathrm{N}(2)$ & $2.019(4)$ \\
\hline $\mathrm{N}(3)-\mathrm{Cu}(1)-\mathrm{N}(2)$ & $90.70(15)$ & $\mathrm{N}(3) \# 2-\mathrm{Cu}(1)-\mathrm{N}(2)$ & $89.30(15)$ \\
\hline $\mathrm{N}(3) \# 2-\mathrm{Cu}(1)-\mathrm{N}(3)$ & \multicolumn{3}{|l|}{180.0} \\
\hline \multicolumn{4}{|c|}{$\begin{array}{c}\text { Symmetry transformations used to generate equivalent atoms for } 1 \text { : } \# 2-\mathrm{x}+2,-\mathrm{y},-\mathrm{z}+1 \\
\text { Compound } \mathbf{2}\end{array}$} \\
\hline $\mathrm{Cu}(1)-\mathrm{N}(1)$ & $2.017(5)$ & $\mathrm{Cu}(1)-\mathrm{N}(4)$ & $2.010(5)$ \\
\hline $\mathrm{Cu}(1)-\mathrm{N}(1)$ & $2.810(2)$ & $\mathrm{N}(4) \# 5-\mathrm{Cu}(1)-\mathrm{N}(1)$ & $90.5(2)$ \\
\hline $\mathrm{N}(4) \# 4-\mathrm{Cu}(1)-\mathrm{N}(4) \# 5$ & $180.0(4)$ & $\mathrm{N}(4) \# 4-\mathrm{Cu}(1)-\mathrm{N}(1)$ & $89.5(2)$ \\
\hline \multicolumn{4}{|c|}{$\begin{array}{l}\text { Symmetry transformations used to generate equivalent atoms for } 2 \text { : \# } 4-y+1 / 2, x-1,-z+1 / 2 \# 5 \\
y+1 / 2,-x+1, z+1 / 2 \text {. }\end{array}$} \\
\hline \multicolumn{4}{|c|}{ Compound $\mathbf{3}$} \\
\hline $\operatorname{Ag}(1)-\mathrm{N}(2)$ & $2.135(5)$ & $\operatorname{Ag}(1)-N(8)$ & $2.139(5)$ \\
\hline $\mathrm{N}(8)-\operatorname{Ag}(1)-\mathrm{N}(2)$ & $179.4(2)$ & & \\
\hline \multicolumn{4}{|c|}{ Compound 4} \\
\hline $\operatorname{Ag}(1)-\mathrm{N}(7)$ & $2.113(11)$ & $\operatorname{Ag}(2)-O(5 W)$ & $2.465(14)$ \\
\hline $\operatorname{Ag}(1)-\mathrm{N}(6)$ & $2.121(12)$ & $\operatorname{Ag}(2)-\operatorname{Ag}(3)$ & $2.812(3)$ \\
\hline $\operatorname{Ag}(1)-O(10)$ & $2.577(11)$ & $\operatorname{Ag}(3)-\mathrm{O}(35)$ & $2.123(11)$ \\
\hline $\operatorname{Ag}(2)-O(33)$ & $2.187(11)$ & $\operatorname{Ag}(3)-O(32)$ & $2.148(10)$ \\
\hline $\operatorname{Ag}(2)-O(34)$ & $2.228(11)$ & $\mathrm{O}(34)-\mathrm{Ag}(2)-\mathrm{O}(5 \mathrm{~W})$ & $92.4(5)$ \\
\hline N(7)-Ag(1)-N(6) & $176.7(5)$ & $\mathrm{O}(33)-\operatorname{Ag}(2)-\operatorname{Ag}(3)$ & $79.6(3)$ \\
\hline $\mathrm{N}(7)-\mathrm{Ag}(1)-\mathrm{O}(10)$ & $89.7(4)$ & $\mathrm{O}(34)-\operatorname{Ag}(2)-\operatorname{Ag}(3)$ & $81.5(3)$ \\
\hline $\mathrm{N}(6)-\mathrm{Ag}(1)-\mathrm{O}(10)$ & $87.3(4)$ & $\mathrm{O}(5 \mathrm{~W})-\operatorname{Ag}(2)-\operatorname{Ag}(3)$ & $172.6(4)$ \\
\hline $\mathrm{O}(33)-\mathrm{Ag}(2)-\mathrm{O}(34)$ & $160.0(5)$ & $\mathrm{O}(35)-\mathrm{Ag}(3)-\mathrm{O}(32)$ & $162.4(5)$ \\
\hline $\mathrm{O}(33)-\mathrm{Ag}(2)-\mathrm{O}(5 \mathrm{~W})$ & $106.9(5)$ & $\mathrm{O}(35)-\mathrm{Ag}(3)-\operatorname{Ag}(2)$ & $82.5(3)$ \\
\hline
\end{tabular}




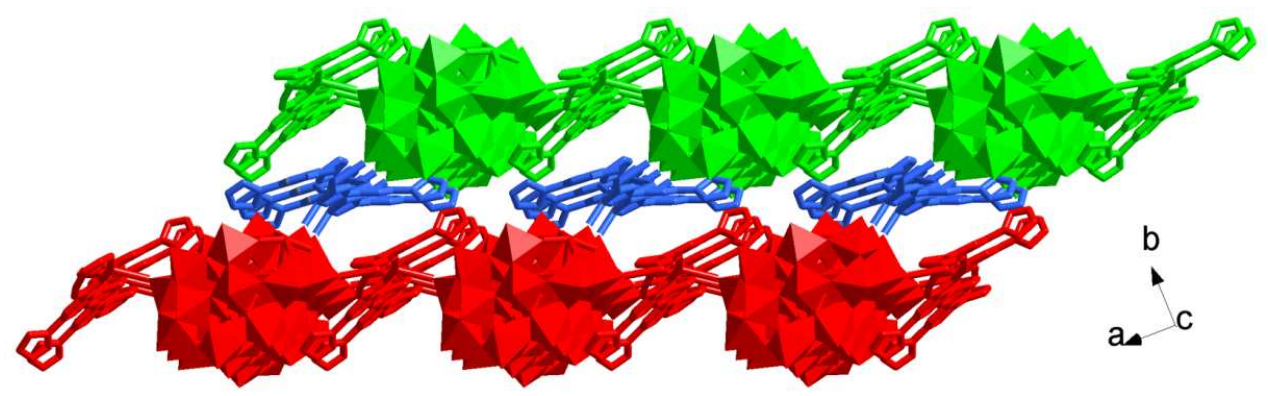

Figure S1. View of the 3D framework constructed from 2D networks and 1D $\left\{\mathrm{Ag}_{3}(\mathrm{HDIBA})_{2}\right\}_{\mathrm{n}}$ chains in compound 4 .
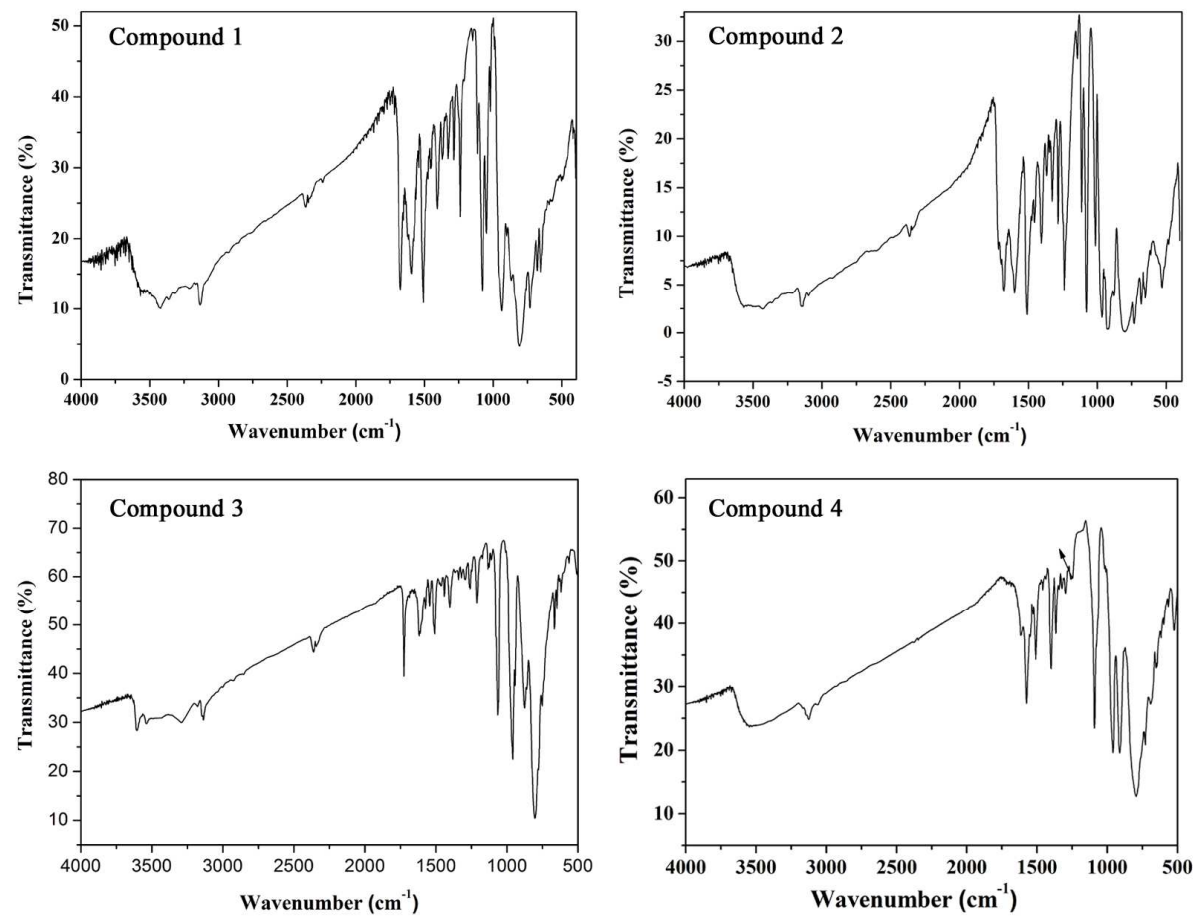

Figure S2. The IR spectra of compounds 1-4. 

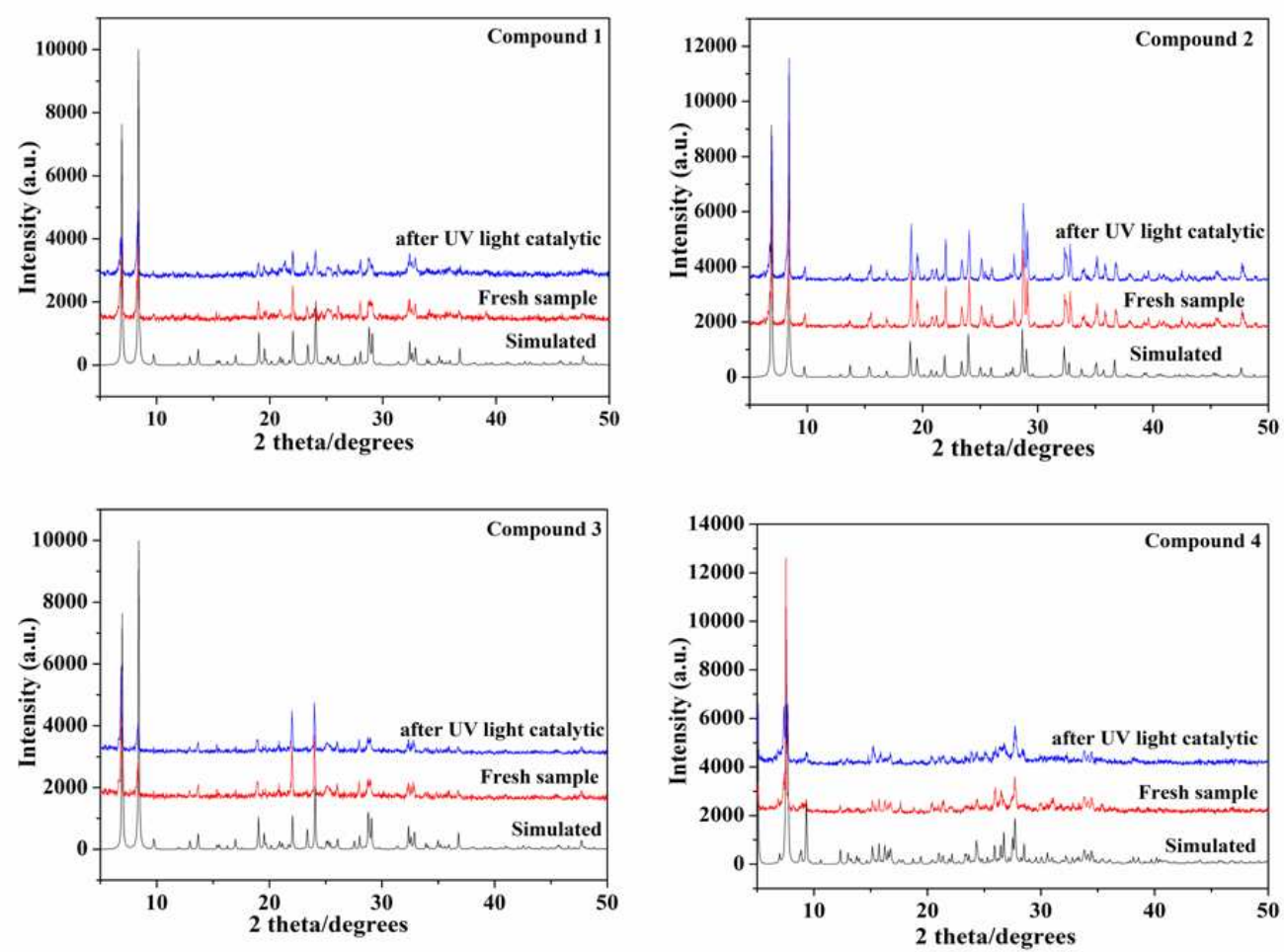

Figure S3. The PXRD patterns for compounds 1-4.

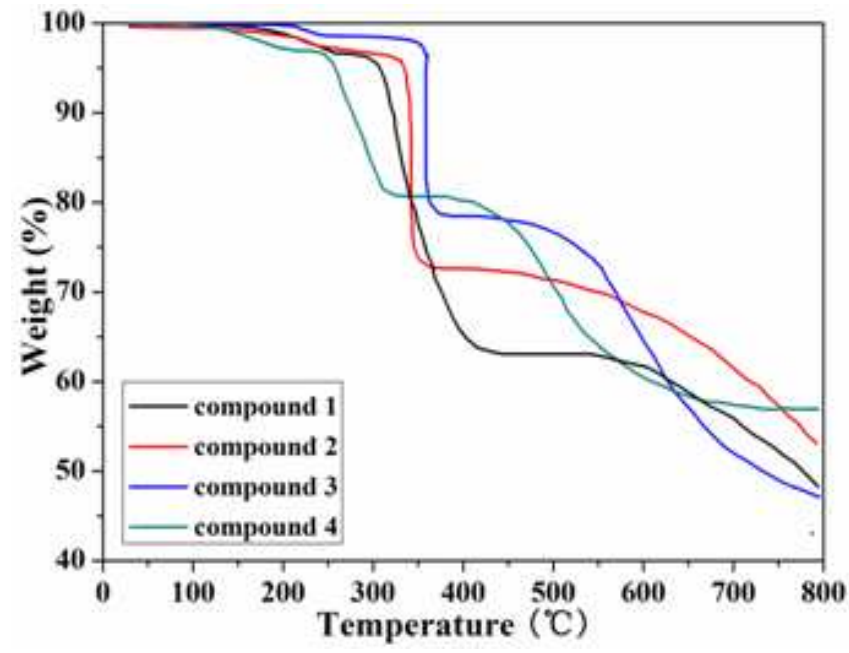

Figure S4. The TG curves of compounds 1-4. 

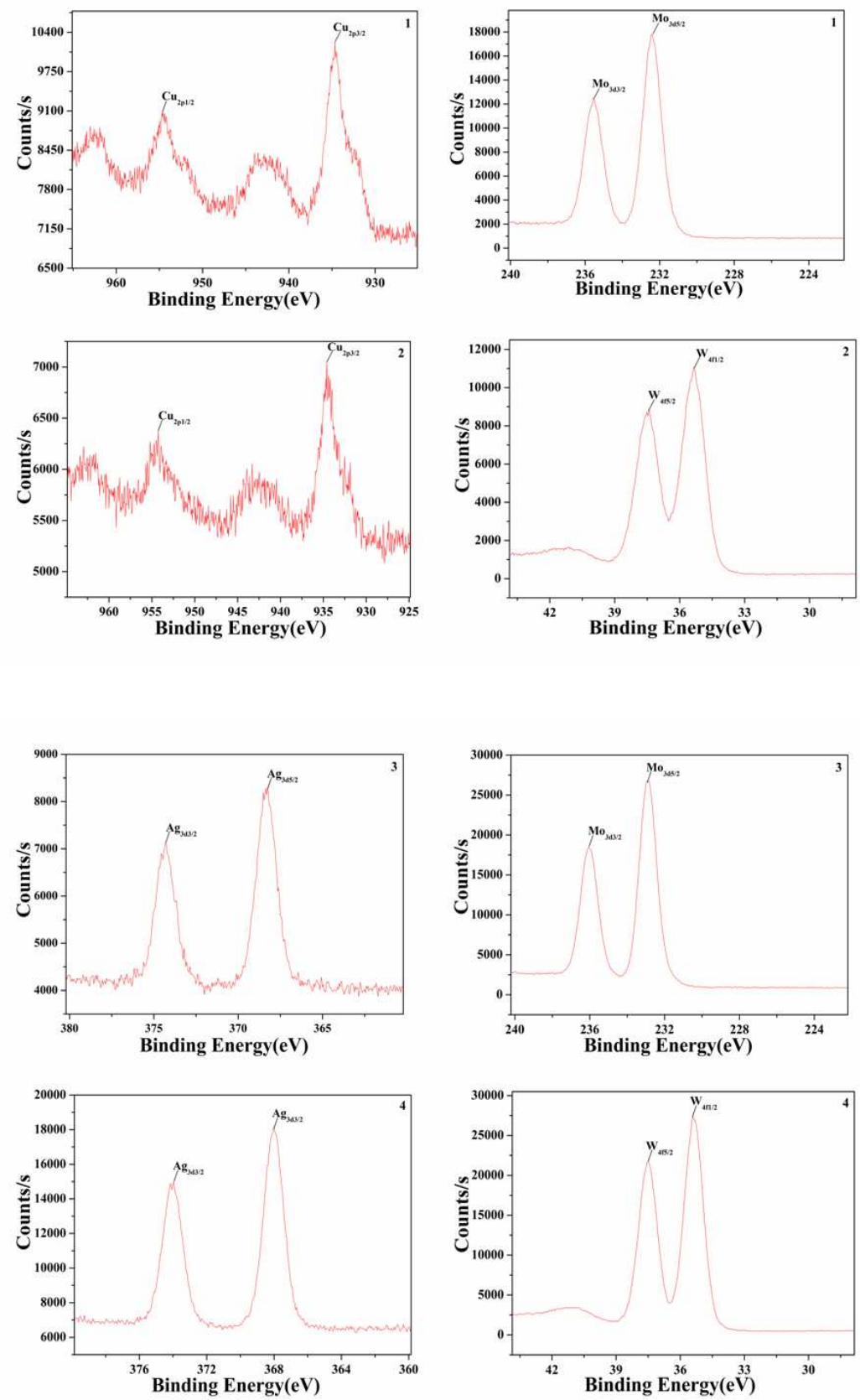

Figure S5. The XPS spectra of compounds 1-4. 


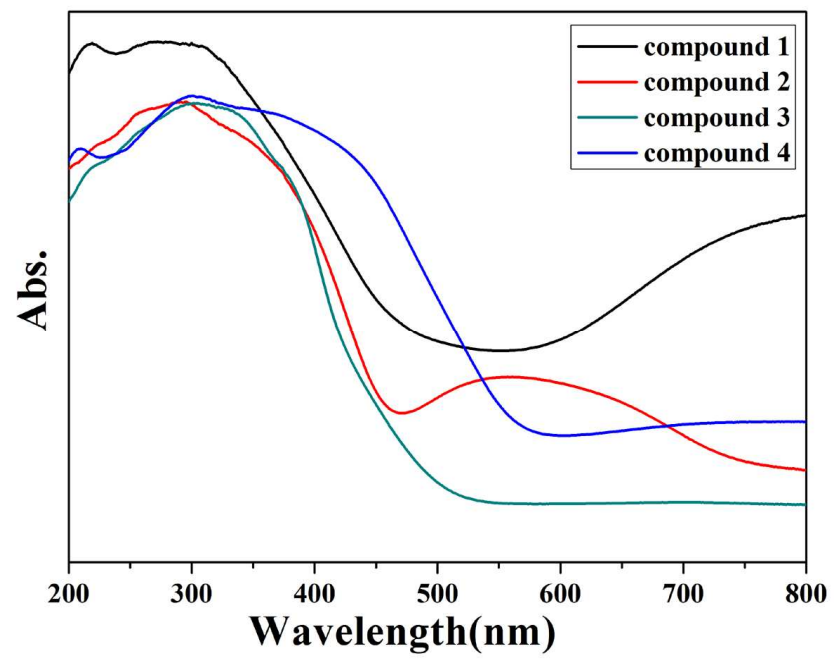

Figure S6. The diffuse reflection spectra of compounds 1-4 in the crystalline state.

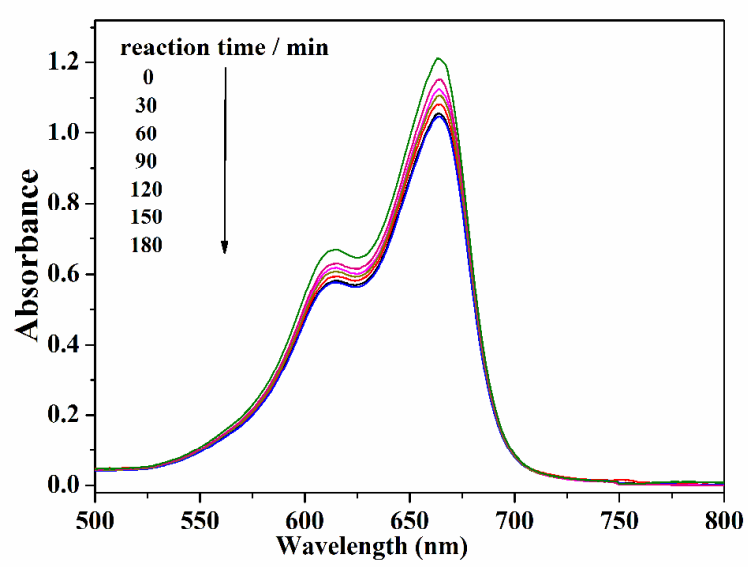

Figure S7. The absorption spectra of the MB solution under UV irradiation without photocatalyst. 

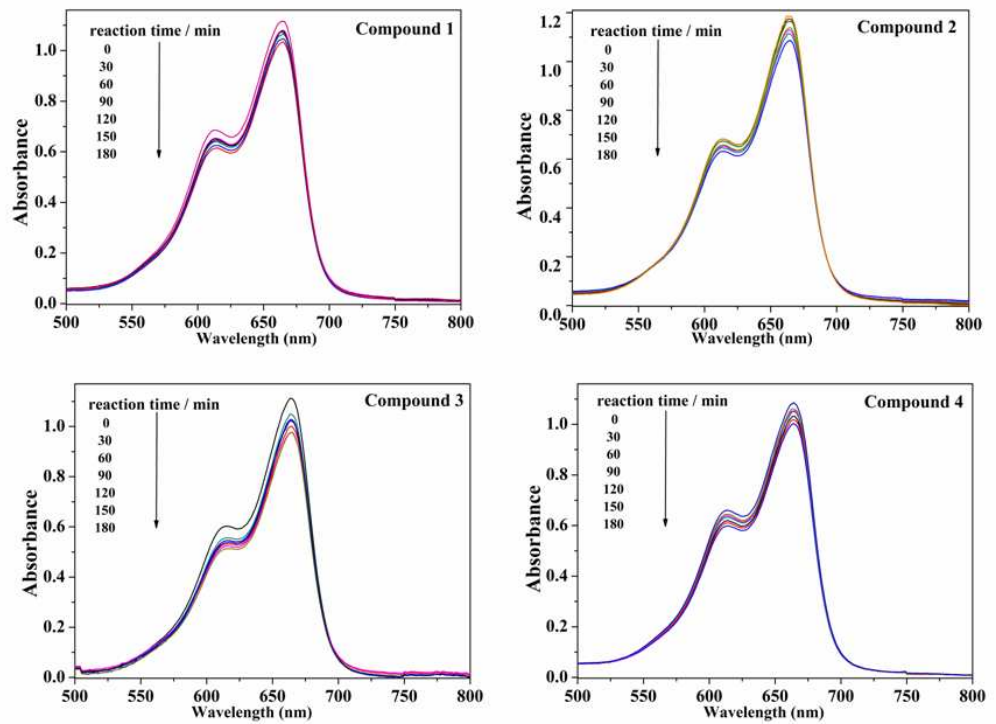

Figure S8. Absorption spectra of the MB solution in dark at the presence of compounds 1-4. 